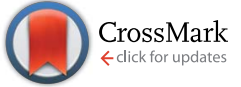

Cite this: RSC Adv., 2017, 7, 10855

Received 30th December 2016 Accepted 4th February 2017

DOI: 10.1039/c6ra28854e

rsc.li/rsc-advances

\section{Hybrid-structured ZnO thermoelectric materials with high carrier mobility and reduced thermal conductivity}

\author{
Dai-Bing Zhang, He-Zhang Li, Bo-Ping Zhang, * Dou-dou Liang and Min Xia
}

Nanostructure engineering has been extensively applied to $\mathrm{ZnO}$ in an effort to improve its performance in thermoelectric material, solar cell, and nanogenerator applications. Nano-structured ZnO bulks are limited by their inherently low mobility caused by the high density of grain boundaries and interfaces. In this study, a hybrid micro/nano structure composed of nearly coherent grain boundaries with a low misorientation degree among the nanograins was successfully fabricated in $\mathrm{Zn}_{1-x} \mathrm{Al} x \mathrm{O}(x=0,0.01,0.02,0.03,0.04$ mol) bulks via hydrothermal synthesis and spark plasma sintering. Despite the large amount of nanograin boundaries and interfaces in the resulting material, a high carrier mobility value $\left(50.7 \mathrm{~cm}^{2} \mathrm{~V}^{-1} \mathrm{~s}^{-1}\right)$ was obtained in the $x=0.2$ sample - close to the level shown by ZnO single crystals and far higher than that of its ordinary nano-structured counterparts $\left(<15 \mathrm{~cm}^{2} \mathrm{~V}^{-1} \mathrm{~s}^{-1}\right)$. A reduced thermal conductivity value of $2.1 \mathrm{~W} \mathrm{~m}^{-1} \mathrm{~K}^{-1}$ at $1073 \mathrm{~K}$ was also obtained in the micro/nano-structured $x=0.02$ bulk due to extremely effective scattering at boundaries and interfaces also present in the nano-structured counterparts. After the simultaneous optimization of both electrical and thermal transport properties, the micro/nanostructured $x=0.02$ sample showed a high $Z T$ value (up to 0.36 ) at $1073 \mathrm{~K}$. The proposed micro/nanostructure may also be applicable to other thermoelectric materials for further $Z T$ enhancement.

\section{Introduction}

Thermoelectric (TE) materials are capable of direct conversion between heat and electricity, which makes them favorable for a number of versatile applications in both power generation and electronic cooling devices. ${ }^{1}$ The efficiency of a TE device is dependent on its material properties, which are characterized by the dimensionless figure of merit $(Z T) ; Z$ is the ratio $\sigma \alpha^{2} / \kappa$ (where $\sigma$ is the electrical conductivity, $\alpha$ is the Seebeck coefficient, $\sigma \alpha^{2}$ is the power factor (PF), and $\kappa$ is the thermal conductivity) and $T$ is the working temperature in kelvin. Good TE performance requires highly efficient electrical transport but low thermal transport properties, ${ }^{2-4}$ though they are intrinsically interdependent and it is very challenging to optimize them synergistically.

$\mathrm{ZnO}$ is well known as a conductive oxide with a wide forbidden bandgap of $3.5 \mathrm{eV}$. The electrical properties of $\mathrm{ZnO}$ have garnered considerable research interest in many fields related to chemistry and physics; in regards to engineering solar cells $^{5,6}$ and nanogenerators, ${ }^{7,8}$ for example. Since the pioneering TE properties of $\mathrm{ZnO}$-based ceramics were first reported by Ohtaki and Tsubota ${ }^{\mathbf{9 1 0}}$ in an Al-doped $\mathrm{ZnO}$, they have been of particular interest as high-temperature $(\geq 1273 \mathrm{~K})$ TE materials

Beijing Key Lab of New Energy Materials and Technology, School of Materials Science and Engineering, University of Science and Technology Beijing, Beijing 100083, China. E-mail: bpzhang@ustb.edu.cn; Tel: +86-10-62334195 due to their low toxicity, high thermal stability, and high electrical conductivity. A secondary phase such as $\mathrm{ZnAl}_{2} \mathrm{O}_{4}$ with an intrinsically high resistance typically appears in Al-doped ceramics due to the low solubility limit of $\mathrm{Al}$ in $\mathrm{ZnO},{ }^{\mathbf{9 , 1 0}}$ which slows the electron transport. The relatively high $\kappa$ value $\sim 5 \mathrm{~W}$ $\mathrm{m}^{-1} \mathrm{~K}^{-1}$ at $1273 \mathrm{~K}$ for Al-doped $\mathrm{ZnO}^{10}$ is another factor limiting any further improvement in TE conversion.

Numerous researchers have attempted to engineer nanostructures to obtain a low $\kappa$ in Al-doped $\mathrm{ZnO}$ ceramics. ${ }^{\mathbf{1 1 - 1 4}}$ Nam et al., for example, applied thermal decomposition and spark plasma sintering (SPS) to prepare a $\mathrm{Zn}_{0.98} \mathrm{Al}_{0.02} \mathrm{O}$ nanostructured bulk sample and achieved a $\kappa$ value as low as $2.0 \mathrm{~W}$ $\mathrm{m}^{-1} \mathrm{~K}^{-1}$ at $1073 \mathrm{~K}$ by virtue of strong phonon scattering caused by the finer grains (average size: $c a .200 \mathrm{~nm}$ ) and $\mathrm{ZnAl}_{2} \mathrm{O}_{4}$ nanoscale precipitates (average size: $c a$. $100 \mathrm{~nm}$ ); $Z T$ value reached 0.34 at $1073 \mathrm{~K}^{12}$ Nano-structured Al-doped $\mathrm{ZnO}$ features low phonon conduction due to the extremely effective scattering facilitated by highly dense grain boundaries and interfaces, but has less favorable electron transport behavior due to low carrier mobility below $15 \mathrm{~cm}^{2} \mathrm{~V}^{-1} \mathrm{~s}^{-1}$ at room temperature ${ }^{11-13}$ - another drawback which substantially limits any further enhancement of TE performance.

In the present study, we were able to create a unique micro/ nano-structure via hydrothermal method. Hydrothermal synthesis (HS) readily facilitates uniform doping and precise control over the size and morphology of powder products with various morphologies such as nanoparticle, ${ }^{15}$ anisotropic 
rod, ${ }^{16,17}$ flower, ${ }^{18,19}$ and others. The nanostructure features must be retained in the bulk materials throughout this process, for which spark plasma sintering (SPS) is often used. SPS enables densification at a low temperature within a short sintering time and allows the solid solubility of dopants and micro/nanostructure of the precursor powders to be retained or frozen in the sintered body. Below, we report the formation of micro/ nano-structured $\mathrm{Zn}_{1-x} \mathrm{Al}_{x} \mathrm{O}(x=0,0.01,0.02,0.03,0.04 \mathrm{~mol})$ bulk materials created by a combination of HS and SPS. As opposed to the misaligned grain boundaries in traditional nano-structured bulks, ${ }^{\mathbf{1 1 - 1 4}}$ the nearly coherent grain boundary among the nanograins attained in our micro/nano-structured bulks facilitated a fast path for electron transport and resulted in carrier mobility almost as high as that of $\mathrm{ZnO}$ single crystals. We also obtained low phonon conduction in the sample materials due to effective scattering at boundaries and interfaces in (most likely) the nano-structured bulks. In short, the proposed micro/nano-structure strategy allowed us to successfully and synergistically optimize both electrical and thermal transport properties, creating a novel approach to $Z T$ enhancement in TE-based, Al-doped $\mathrm{ZnO}$ ceramics.

\section{Experimental procedure}

\section{Synthesis of micro/nano-structured powders and bulk samples}

$\mathrm{Zn}_{1-x} \mathrm{Al}_{x} \mathrm{O}(x=0,0.01,0.02,0.03,0.04 \mathrm{~mol})$ micro/nanostructured powders were prepared by HS using zinc and aluminum acetates as raw materials and triethanolamine (TEA) as a surfactant. The HS was performed at $453 \mathrm{~K}$ in a solution with $\mathrm{pH}$ fixed at 7.0-8.0. The resultant white solid product was filtered, washed with de-ionized water and absolute ethyl alcohol several times, and dried at $353 \mathrm{~K}$ in an oven for $10 \mathrm{~h}$ in air. The dried powders were then sintered in a $\Phi 20 \mathrm{~mm}$ graphite mold under an axial compressive stress of $50 \mathrm{MPa}$ at $1173 \mathrm{~K}$ in vacuum in a commercial SPS system (Sumitomo SPS1050, Japan) at ramp-up speed of $100 \mathrm{~K} \mathrm{~min}^{-1}$ and $5 \mathrm{~min}$ holding time. The samples were disk-shaped bulks $\Phi 20 \mathrm{~mm} \times 10 \mathrm{~mm}$ in size.

\section{Characterization}

Phase structure was identified by X-ray diffraction with $\mathrm{Cu} \mathrm{K} \alpha$ radiation $(\lambda=1.5406 \AA$ ) filtered through Ni foil (Rigaku; RAD-B system, Tokyo, Japan). The lattice parameters were determined by least squares method in Jade 5 software. The morphologies of powders, fractographs of bulks, and elemental distribution in the nanograins and nanoprecipitates were investigated with a field emission scanning electron microscope (FESEM, ZEISS SUPRA ${ }^{\mathrm{TM}}$ 55, Germany) equipped with a Kevex model 3200 energy dispersive X-ray (EDX) spectroscope. The crystallographic direction was investigated with a high resolution transmission electron microscope (HRTEM, Philips Tecnai F20, Holland).

\section{Thermoelectric measurements}

The carrier concentration $(n)$ and carrier mobility $(\mu)$ at room temperature were measured with a DC-8340 apparatus (TOYO, Japan). The Seebeck coefficient and electrical conductivity were measured at $323-1073 \mathrm{~K}$ in a helium atmosphere on a Seebeck coefficient/electric resistance measured system (ZEM-2, UlvacRiko, Japan). The thermal conductivity $(\kappa)$ was calculated from the product of thermal diffusivity $(D)$, specific heat $\left(C_{\mathrm{p}}\right)$, and density $(d), \kappa=D C_{\mathrm{p}} d$. The thermal diffusivity $D$ was measured at 323-1073 K by laser flash method with a TC-9000 apparatus (Ulvac-Riko, Japan). The specific heat $C_{\mathrm{p}}$ was measured at 323$1073 \mathrm{~K}$ using a DSC-60 apparatus (SHIMADZU, Japan). All samples were measured perpendicular to the pressing direction.

\section{Results and discussion}

\section{Thermoelectric properties of micro/nano structured $\mathrm{ZnO}$ - based bulks}

Fig. 1 shows the TE properties of our micro/nano-structured $\mathrm{Zn}_{1-x} \mathrm{Al}_{x} \mathrm{O}(x=0,0.01,0.02,0.03,0.04 \mathrm{~mol})$ bulks. For comparison, previously reported data ${ }^{11-13}$ for $\mathrm{Zn}_{1-x} \mathrm{Al}_{x} \mathrm{O}$ bulks with grain size of $c a .200 \mathrm{~nm}$ are included; these are defined as "nanostructured bulks" to distinguish them from our micro/nanostructured bulks. As shown in Fig. 1a, the micro/nano-structured $x=0.02$ bulk sample reached the optimized $Z T$ value, 0.36 , at $1073 \mathrm{~K}$, which is lower than the $Z T$ value of 0.44 , at $1000 \mathrm{~K}$, reproduced from Jood et al. in $\mathrm{Zn}_{0.9975} \mathrm{Al}_{x} \mathrm{O}$ nano-structured bulks. It has been well established that $Z T_{\text {ave }}$ (not $Z T_{\max }$ ) over the entire working temperature range determines the TE conversion efficiency, as-calculated by $Z T_{\text {ave }}=\frac{1}{T_{\mathrm{h}}-T_{\mathrm{c}}} \int_{T_{\mathrm{c}}}^{T_{\mathrm{h}}} Z T \mathrm{~d} T$ (where $T_{\mathrm{h}}$ and $T_{\mathrm{c}}$ are the temperature at the hot-side and cold-side, respectively ${ }^{20}$ ). In the temperature range of $373-1073 \mathrm{~K}$, the $Z T_{\text {ave }}$ of the micro/ nano-structured $x=0.02$ bulk was $c a$. 0.13 - again, higher than that of the nano-structured bulks (Fig. 1b), suggesting that a higher TE conversion efficiency was achieved in the micro/nano structured $x=0.02$ bulk.

Any improvement in $Z T_{\text {ave }}$ value relies on a simultaneous enhancement of several TE parameters. The thermal conductivity $(\kappa)$ shown in Fig. 1c for all the micro/nano-structured bulks continuously decreased over the measuring temperature range, indicating a dominant phonon scattering mechanism. In addition, $\kappa$ decreased with $x$ down to $1.6 \mathrm{~W} \mathrm{~m}^{-1} \mathrm{~K}^{-1}$ at its lowest point in the micro/nano-structured $x=0.04$ bulk at $1073 \mathrm{~K}$. At the same $x=0.02$ composition, the micro/nano-structured bulk showed a lower $\kappa$ value $\left(2.1 \mathrm{~W} \mathrm{~m}^{-1} \mathrm{~K}^{-1}\right)$ than its counterparts at $1073 \mathrm{~K}$, comparable to $1.9 \mathrm{~W} \mathrm{~m}^{-1} \mathrm{~K}^{-1}$ (ref. 12 ) and $3.2 \mathrm{~W} \mathrm{~m}^{-1}$ $\mathrm{K}^{-1}$ (ref. 13) in the nano-structured bulk samples; this indicates that low phonon conduction was also effectively retained in the micro/nano-structured bulk due to extremely effective scattering on boundaries and interfaces (which likely were present in the nano-structured bulks).

In regards to electrical transport properties, we observed an outstanding $\sigma$ value of $1270 \mathrm{~S} \mathrm{~cm}^{-1}$ in the micro/nanostructured $x=0.02$ bulk at room temperature (Fig. 1d) - far higher than the $\sigma$ value of $50-200 \mathrm{~S} \mathrm{~cm}^{-1}$ in the nano-structured bulks. The negative Seebeck coefficient $(\alpha)$ shown in Fig. 1e indicated that all samples are n-type semiconductors regardless of the differences in microstructure among them. The $|\alpha|$ values of $45.5-59.8 \mu \mathrm{V} \mathrm{K}^{-1}$ were obtained at room temperature in the 

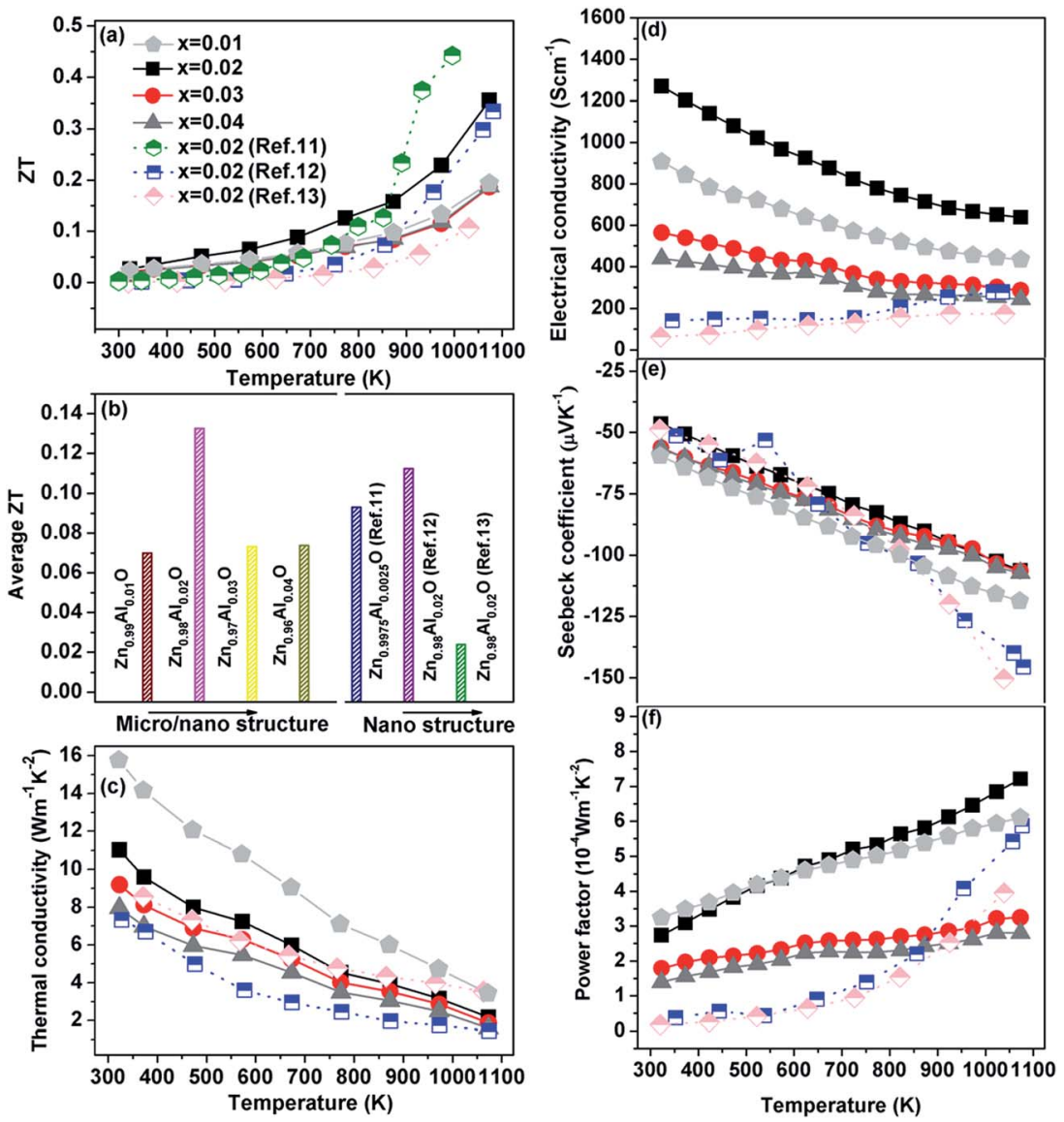

Fig. 1 Temperature dependence of the $Z T$ value (a), thermal conductivity (c), electrical conductivity (d), Seebeck coefficient (e), and power factor (f) for $\mathrm{Zn}_{1-x} \mathrm{Al}_{x} \mathrm{O}\left(x=0.01,0.02,0.03,0.04 \mathrm{~mol}\right.$ ) bulks. Note: image (b) is $Z T_{\text {ave }}$ in the temperature range of $373-1073 \mathrm{~K}$. The data of nanostructured bulks from the ref. 11-13 are given for comparison.

micro/nano-structured bulks, they are comparable to the $|\alpha|$ of ca. $50 \mu \mathrm{V} \mathrm{K} \mathrm{K}^{-1}$ for the nano-structured counterparts. The outstanding $\sigma$ value combined with the comparable $|\alpha|$ value contributed to the final enhanced power factor $(\mathrm{PF})$ shown in Fig. 1f: the highest $\mathrm{PF}$ value, $7.2 \times 10^{-4} \mathrm{~W} \mathrm{~m}^{-1} \mathrm{~K}^{-2}$, was obtained at $1073 \mathrm{~K}$ in the micro/nano-structured $x=0.02$ bulk and was far higher than that of any nano-structured bulk (especially at the low and medium temperatures).

These results altogether indicate that we did indeed synergistically optimize both the electrical and thermal transport properties in our micro/nano-structured bulk samples. Low phonon conduction can be successfully achieved by ensuring effective scattering from the highly dense grain boundaries and interfaces, though as mentioned in the Introduction, it is very challenging to maintain favorable electrical transport properties. The unique phenomenon we observed in our samples, especially in regards to their electrical transport properties, will be discussed as related to the novel micro/nano-structure in greater detail below.

Because $\sigma$ value is expressed by $\sigma=n e \mu$, (where $n$ and $\mu$ are the carrier concentration and the carrier mobility, respectively) the outstanding $\sigma$ we observed in our micro/nano-structured bulks is attributable to high $n$ and $\mu$ values. The Hall measurement results shown in Fig. 2 a indicated that the $n$ value increased dramatically from $1.4 \times 10^{18} \mathrm{~cm}^{-3}$ to $8.5 \times 10^{19} \mathrm{~cm}^{-3}$ and $1.5 \times 10^{20} \mathrm{~cm}^{-3}$, as $x=0$ increased to $x=0.01$ and $x=0.02$. This occurred mainly due to the formation of point-defects such as $\mathrm{Al}_{\mathrm{i}}^{\cdots}$ and/or $\mathrm{Al}_{\mathrm{Zn}}^{.}$ following the reactions $\mathrm{Al}_{2} \mathrm{O}_{3} \stackrel{\mathrm{ZnO}}{\longrightarrow} 2 \mathrm{Al}_{\mathrm{i}}{ }^{\cdots}+3 \mathrm{O}_{\mathrm{O}}^{\times}+6 \mathrm{e}^{\prime}$ and $\mathrm{Al}_{2} \mathrm{O}_{3} \stackrel{\mathrm{ZnO}}{\longrightarrow} 2 \mathrm{Al}_{\mathrm{Zn}}^{\circ}+3 \mathrm{O}_{\mathrm{O}}+2 \mathrm{e}^{\prime}$. The former interstitial solution of $\mathrm{Al}^{3+}$ enlarged the $\mathrm{ZnO}$ lattice, while the latter substitution solution of $\mathrm{Al}^{3+}$ for $\mathrm{Zn}^{2+}$ shrank the lattice due to the smaller ion radius $(0.49 \AA)$ of $\mathrm{Al}^{3+}$ compared to $\mathrm{Zn}^{2+}(0.74 \AA) .{ }^{17}$

The lattice parameters $(a=b=3.259 \AA, c=5.217 \AA)$ of our $x$ $=0.02$ bulk sample were almost unchanged compared to the parameters $(a=b=3.260 \AA, c=5.215 \AA)$ of the $x=0$ bulk, which may be ascribed to the fact that $\mathrm{Al}_{\mathrm{Zn}}$ and $\mathrm{Al}_{\mathrm{i}}^{\cdots}$ co-existed in the $x=0.02$ bulk; the influence of the former on the lattice parameters offset those of the latter. As $x$ increased to 0.03 and $0.04, n$ value decreased slightly but stayed around $c a .1 \times 10^{20}$ $\mathrm{cm}^{-3}$, suggesting that the $\mathrm{Al}^{3+}$ solution in the $\mathrm{ZnO}$ neared saturation as $x$ reached $0.02 .^{10}$ The $\mu$ value also increased from $45.5 \mathrm{~cm}^{2} \mathrm{~V}^{-1} \mathrm{~s}^{-1}$ to $54.2 \mathrm{~cm}^{2} \mathrm{~V}^{-1} \mathrm{~s}^{-1}$ as $x=0$ increased to 0.01 , apart from a slight decrease in $\mu$ value to $50.7 \mathrm{~cm}^{2} \mathrm{~V}^{-1} \mathrm{~s}^{-1}, 40.9$ $\mathrm{cm}^{2} \mathrm{~V}^{-1} \mathrm{~s}^{-1}$ and $30.5 \mathrm{~cm}^{2} \mathrm{~V}^{-1} \mathrm{~s}^{-1}$ at $x=0.02, x=0.03$ and $x=$ 0.04 , respectively. Generally, the $\mu$ should decrease after doping $\mathrm{Al}^{3+}$, according to reason that the high $n$ value especially in the 

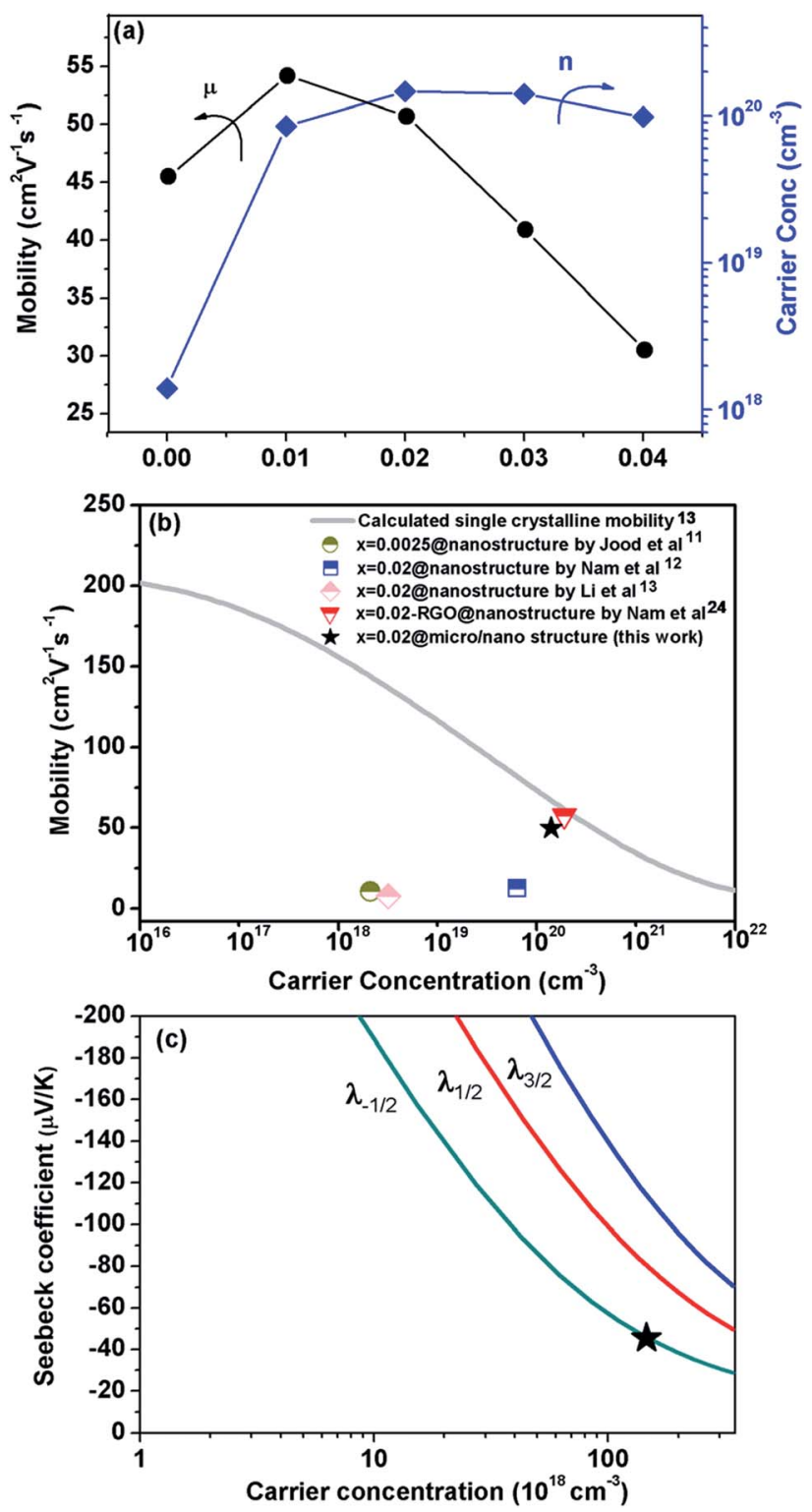

Fig. 2 (a) Carrier concentration ( $n$ ) and carrier mobility $(\mu)$ at room temperature in micro/nano-structured $\mathrm{Zn}_{1-x} \mathrm{Al}_{x} \mathrm{O}(x=0.01,0.02,0.03$, 0.04 mol) bulks; (b) relationship between $n$ and $\mu$ at room temperature for micro/nano structured $x=0.02$ bulk, in which the data of $\mathrm{ZnO}$ single crystal calculated based on BHD model ${ }^{24}$ by a gray line and nanostructured $\mathrm{Zn}_{1-x} \mathrm{Al}_{x} \mathrm{O}$ without ${ }^{11-13}$ and with $\mathrm{RGO}^{24}$ are given for comparison; (c) relationship between Seebeck coefficient and $n$ for micro/nano structured $x=0.02$ bulk, with three lines calculated according to $\lambda=1 / 2,3 / 2$, and $-1 / 2$ denotes electron scattering from optical phonons, ionized impurities, and acoustic phonons, respectively.

high level of $n \geq 10^{20} \mathrm{~cm}^{-3}$ increases significantly due to ionized impurity scattering. ${ }^{21}$ The secondary $\mathrm{ZnAl}_{2} \mathrm{O}_{4}$ usually appears as $x \geq 0.02$ and forms a large amount of $\mathrm{ZnO} / \mathrm{ZnAl}_{2} \mathrm{O}_{4}$ interfaces, which should also increase electron scattering and decrease $\mu$ value. ${ }^{11}$ Regardless of the high $n$ value (up to $8.5 \times 10^{19} \mathrm{~cm}^{-3}$ ) we observed an unexpected increase in $\mu$ at $x=0.01$ and a subsequent decrease; these phenomena attributed to $x$ are likely related to the novel micro/nano-structure, which will be discussed in detail later in this paper.
The relationship between $n$ and $\mu$ at room-temperature for micro/nano structured $x=0.02$ bulk and nanostructured $\mathrm{Zn}_{1-x} \mathrm{Al}_{x} \mathrm{O}$ bulks without ${ }^{11-13}$ and with $\mathrm{RGO}^{24}$ is plotted in Fig. $2 \mathrm{~b}$. The micro/nano-structured $x=0.02$ bulk showed higher $n$ and $\mu$ values than the nanostructured bulks, ${ }^{11-13}$ indicating that either high solid solubility of $\mathrm{Al}^{3+}$ or fast electron transport was realized in the micro/nano-structured $x=0.02$ bulk. The single crystalline $\mathrm{ZnO}$ is known to have excellent electron transport behavior due to the lack of boundaries and interfaces, whose mobility values are calculated based on Matthiessen's rule and BHD model $\mathrm{l}^{22,23}$ and plotted in Fig. $2 \mathrm{~b}$ by a gray line as a function of $n$ value..$^{24}$ In a recent study, Nam et al. reported that the introduction of reduced graphene oxide (RGO) into nanostructured $x=0.02$ bulk can change the electron transport behavior from nanocrystalline to single crystalline. ${ }^{24}$ When the electrons move across the grain boundaries as freely as they are in the single crystalline $\mathrm{ZnO}$, the $\mu$ value is not significantly affected by the grain boundaries due to the reduced or eliminated Schottky barriers. Adding $1 \mathrm{wt} \%$ RGO contributed here to relatively high $\mu$ value, $57.0 \mathrm{~cm}^{2} \mathrm{~V}^{-1} \mathrm{~s}^{-1}$, in nano-structured $x=$ 0.02 bulk; this is quite compatible with single crystalline $\mathrm{ZnO}^{24}$ Interestingly, the micro/nano-structured $x=0.02$ bulk without RGO also featured a high $\mu$ value $\left(50.7 \mathrm{~cm}^{2} \mathrm{~V}^{-1} \mathrm{~s}^{-1}\right)$, indicating that similar electron transport behavior to that of single crystalline $\mathrm{ZnO}$ was achieved in the micro/nano-structured $x=0.02$ bulk as well.

We utilized eqn (1)-(4) to assess the carrier scattering mechanisms. According to the Pisarenko relation for degenerated semiconductors,

$$
|\alpha|=\frac{8 \pi^{2} k_{\mathrm{B}}^{2} T}{3 q h^{2}} m^{*}\left(\frac{\pi}{3 n}\right)^{2 / 3}
$$

where $k_{\mathrm{B}}$ is the Boltzmann constant, $T$ is the absolute temperature, $h$ is Planck's constant, $q$ is an electron charge, and $m^{*}$ is the DOS effective mass at the Fermi level. By plotting the room temperature $\alpha$ vs. $n^{-2 / 3},{ }^{13}$ the $m^{*}$ of the micro/nano-structured $x$ $=0.02$ bulk was estimated to be $0.58 m_{\mathrm{e}}$. According to the measured $n$ and estimated $m^{*}$ values, a simple parabolic band model can be applied by employing the following equations: ${ }^{25}$

$$
\begin{gathered}
F_{\mathrm{r}}=\int_{0}^{\infty} \frac{x^{\mathrm{r}}}{1+\exp (x-\xi)} \mathrm{d} x \\
n=\frac{4}{\pi}\left(\frac{2 \pi m^{*} k_{\mathrm{B}} T}{h^{2}}\right)^{3 / 2} F_{1 / 2} \\
\alpha=-\frac{k_{\mathrm{B}}}{e}\left[\frac{\left(\frac{5}{2}+\lambda\right) F_{3 / 2+\lambda}}{\left(\frac{3}{2}+\lambda\right) F_{1 / 2+\lambda}}-\xi\right.
\end{gathered}
$$

where $F_{\mathrm{r}}$ is the Fermi integral and $\xi$ is the reduced electrochemical potential. $\lambda$ is a scattering parameter and $-1 / 2$ is assumed for acoustic phonon scattering, 1/2 for optical phonon scattering, and $2 / 3$ for ionized impurity scattering. The calculated $\alpha$ values at room temperature as a function of $n$ value are shown in Fig. 2c, in which three lines correspond to these three types of scattering mechanisms, respectively. The $|\alpha|$ value for 
the micro/nano-structured $x=0.02$ bulk corresponds well with to the calculated value when $\lambda=-1 / 2$, indicating that the acoustic phonon was the dominant scattering mechanism. Accordingly, it is safe to assume that electron transport was strongly scattered by the high density of grain boundaries in the micro/nano-structured $x=0.02$ bulk, which should lead to a drastic decrease in $\mu$ value. That being said, we unexpectedly obtained a higher $\mu$ value of $50.7 \mathrm{~cm}^{2} \mathrm{~V}^{-1} \mathrm{~s}^{-1}$ in the micro/nanostructured $x=0.02$ bulk, which was likely related to the novel micro/nano-structured characteristics.

The novel micro/nano-structured characteristics of $x=0.02$ bulk are depicted in Fig. 3 via the FESEM and HRTEM observations. An abundance of smooth micro-spheres $c a .3 \mu \mathrm{m}$ in diameter were observed in the FESEM micrograph as shown in Fig. 3a; this manner of surface morphology indicates that they are dense aggregates composed of nanograins $c a .200 \mathrm{~nm}$ in size. This novel structure, the so-called "micro/nano-structure" to which we refer in this paper, is dependent on the original structure in the corresponding powders shown in Fig. $3 \mathrm{~b}$ where the nanograins are oriented orderly along the axial direction of the micro-spheres due to the spherical restrictions.

We performed HRTEM and fast Fourier transformation electric diffraction (FFT ED) analyses to examine the misorientation degree among the oriented nanograins in the micro/ nano-structured $x=0.02$ bulk. According to the FFT ED diffractograms (acquired from the yellow regions 1 and 2) shown in Fig. 3c, each nanograin exhibited a nearly identical [0001] zone-axis pattern. There also was similar lattice spacing of $0.28 \mathrm{~nm}$ corresponding to the distance between the (1010) lattice planes of ZnO. The HRTEM image shown in Fig. 3d also indicated clear, uniform stripes (acquired from the yellow region 3) even across the grain boundaries (described by the red lines) among the nanograins. The corresponding FFT ED diffractogram shown in Fig. 3e further confirmed that all nanograins in region 3 exhibited practically identical [0001] zone-axis patterns with misorientation degree of about $c a .4^{\circ}$; in effect, a nearly coherent grain boundary was successfully obtained.

We speculated that the misorientation degree would decreased as the diameter of rod-like and spheroidal particles in the microspheres decreased; to this effect, we assumed that refining the grain sizes would enhance the degree of coherence between grain boundaries and thus the rapid transfer of electrons. This novel micro/nano structure characteristic is similar to the mosaic structure characteristic obtained in $\mathrm{Cu}_{2}(\mathrm{~S}, \mathrm{Te})$ polycrystalline bulks prepared via flash cooling. ${ }^{26}$ Said mosaic structure characteristic functions similarly to quasi-single crystals composed of nanograins with identical (or nearly identical) orientation as electrons are freely transferred along the frames of quasi-single crystals. ${ }^{26}$ Hence, the nearly coherent grain boundary among the nanograins for the micro/nano-structures likely dominated the electron transport behavior (again, like single crystalline $\mathrm{ZnO}$ ) in the $x=0.02$ bulk sample (Fig. 2b).

We further explored the electron transport behavior in our samples by comparison among them, as depicted in Fig. $3 \mathrm{f}$ and $\mathrm{g}$. As opposed to the nanostructure in which nanograins are oriented completely randomly and their misaligned interfaces affect electron transfer (black arrow in Fig. 3f), nanograins in the
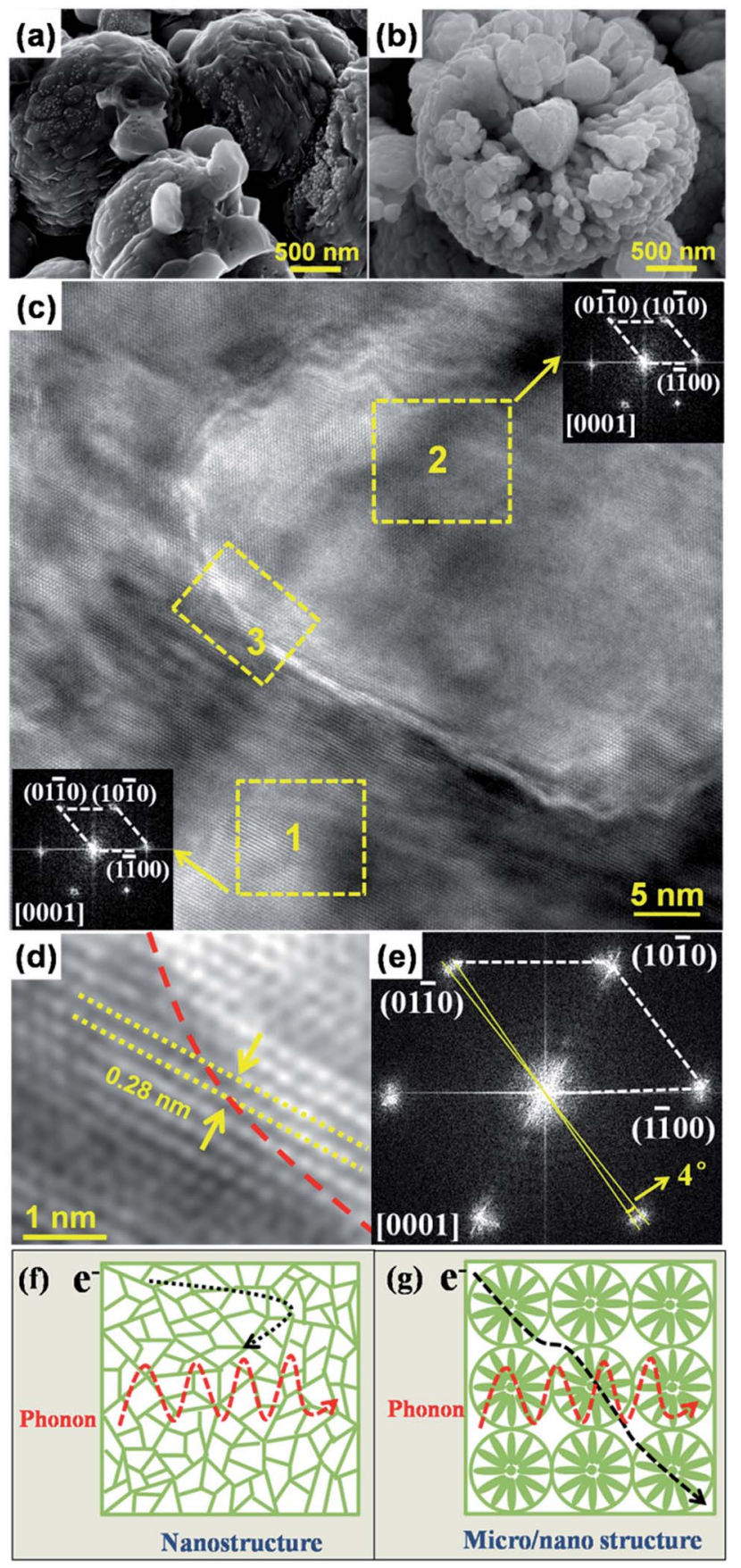

Fig. 3 ( $a$ and $b$ ) FESEM micrograph of micro/nano structured $x=0.02$ bulk (a) and powder (b); (c) HRTEM image and its FFT ED diffractogram of selected regions $(1,2)$ marked with yellow line in micro/nanostructured $x=0.02$ bulk; (d) enlarged HRTEM image of selected region 3 from (c) and FFT ED diffractogram (e), in which the angle between two yellow lines represents degree of misorientation between the two nanograins; structural characteristics of the nanostructure ( $f$ ) and micro/nano-structure (g).

novel micro/nano-structure were orderly oriented along the axial direction of micro-spheres due to the spherical restrictions (Fig. 3g), as discussed above; again, this seemed conducive to obtaining the nearly coherent nanograin boundaries which facilitate a fast path for electron transport. The conjoined micro- 
spheres in the micro/nano-structure also created a coherent crystalline framework for the less-affected electron transport (black arrow in Fig. 3g). In regards to phonon transport, the nanostructure and micro/nano-structure were expected to feature analogous behavior based on the fact that a phonon does not select its own transport path (red arrows in Fig. $3 \mathrm{f}$ and $\mathrm{g}$ ).

These observations altogether again confirm that a novel micro/nano microstructure was successfully obtained in the $x=$ 0.02 bulk sample in which nanograins with nearly coherent grain boundaries not only provided a favorable crystalline framework for rapid electron transfer, but also effectively scattered phonons. This allowed us to synergistically optimize both electrical and thermal transport properties, as depicted in Fig. 1, in the $x=0.02 \mathrm{micro} /$ nano-structured bulk sample; we achieved "phonon-glass electron-crystal" characteristics in our sample, a classic thermoelectric concept. ${ }^{27}$

\section{Shape-controlled synthesis of micro/nano-structured $\mathrm{ZnO}$ - based powders}

The formation of the novel micro/nano-structure in bulk materials depends on the original micro/nano-structure in the corresponding powders, as the precursor powder microstructure is easily retained or frozen in the sintered body during (brief, lowtemperature) SPS. We used triethanolamine (TEA) as a surfactant in preparing the precursor powder via HS. The function of this material on the precursor powder morphology is schematically proposed in Fig. 4. As shown in Fig. 4a, TEA was initially dissociated in water to provide a basic alkalescent environment (eqn (1)) to form a $\mathrm{Zn}(\mathrm{OH})_{2}$ precipitate complex (eqn (2)) which favors the anisotropic growth of $\mathrm{ZnO} .^{16,17}$ Along with TEA chelation, it also coordinated with $\left[\mathrm{Zn}(\mathrm{OH})_{2}\right]_{n}$ to form a complex $\left[\mathrm{Zn}(\mathrm{OH})_{2} \mathrm{TEA}\right]_{n}$ (eqn (3)) which transformed into $\mathrm{ZnO} \cdot n \mathrm{TEA}$ nuclei during the HS process (eqn (4)). The corresponding nucleation process is schematically shown in Fig. 4 b. During the growth process, wurtzite-structured $\mathrm{ZnO}$ tends to take anisotropic growth along the [0001] direction under $\mathrm{OH}^{-}$rich conditions with $\mathrm{NH}_{3} \cdot \mathrm{H}_{2} \mathrm{O}$; $\mathrm{OH}^{-}$united with $\mathrm{Zn}^{2+}$ forms the growth unit $\left[\mathrm{Zn}(\mathrm{OH})_{4}\right]^{2-}$ dominating the anisotropic growth of $\mathrm{ZnO} .^{16,17}$ In our experiment, the anisotropic growth of $\mathrm{ZnO}$ particles proceeded under the dominant eqn (1) as TEA content was relatively low (Fig. 4c). With moderate TEA content, some TEA molecules that did not participate in eqn (1) were absorbed on the surface of $\mathrm{ZnO}$ particles and limited their anisotropic growth to some extent. With high TEA content, the ZnO particles did not readily refine as their sizes reached the nanoscale, because the TEA absorbed around $\mathrm{ZnO}$ particles and became saturated; at this point, aggregation began to accelerated due to TEA chelation because the abundant hydroxyl groups of the TEA rims absorbed on ZnO nanoparticles interacted with each other. This resulted in linkage or a bridging of the neighboring $\mathrm{ZnO}$ nanoparticles. ${ }^{28}$ The $\mathrm{ZnO}$ nanoparticles also aggregated ultimately into micro-spheres along the axial direction, reducing the surface Gibbs free energy of the system. In short, the orientation of nanoparticles should be spherically restricted rather than randomly self-assembled.

The FESEM micrographs of $\mathrm{ZnO}$ powders with various TEA contents shown in Fig. 5a-e support the above description. At

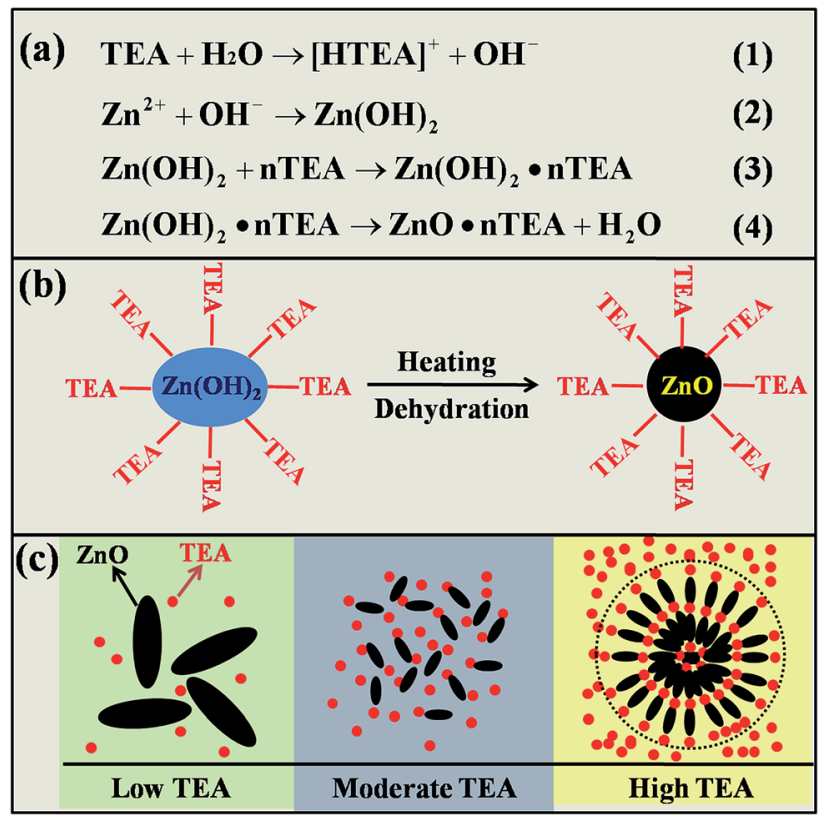

Fig. 4 Function of TEA on preparing micro/nano powders during HS process including reaction equations (a); nucleation (b); growth (c).

TEA content of $5 \mathrm{ml}$ (Fig. 5a), the sample consisted of a single or a twined $\mathrm{ZnO}$ with bullet-like morphology, average length of $c a$. $6 \mu \mathrm{m}$, and mean diameter of $c a .4 \mu \mathrm{m}$ (the aspect ratio is $c a .1 .5$ ), indicating the persistence of anisotropic growth. Well-resolved hexagonal edges and corners were also observed, indicating favorable crystalline $\mathrm{ZnO}$ quality. As TEA content increased to $10 \mathrm{ml}$ (Fig. 5b), mushroom-like ZnO particles with cusped ends formed with a decreased average length of $c a .4 \mu \mathrm{m}$ and mean diameter of $c a .2 \mu \mathrm{m}$ (aspect ratio $=c a .2$ ), indicating that the TEA as surface modifier suppressed growth both in length and in diameter.

Similar inhibition in $\mathrm{ZnO}$ growth has been reported by previous researchers using TEA as a surface modifier. ${ }^{29}$ Others have also confirmed a joint behavior along the radial plane of mushroom-like $\mathrm{ZnO}$ particles resulting from the linkage behavior caused by TEA. ${ }^{29}$ By further increasing TEA to $20 \mathrm{ml}$ (Fig. 5c), spheroidal particles emerged with a decreased average grain size to $c a .600 \mathrm{~nm}$. Interestingly, most spheroidal particles began to aggregate to micro-spheres with an average size of $c a .2$ $\mu \mathrm{m}$ as TEA increased to $25 \mathrm{ml}$ (Fig. 5d). This aggregative behavior was further enhanced by increasing TEA to $30 \mathrm{ml}$, at which point a large amount of micro-spheres $c a .5 \mu \mathrm{m}$ in size were obtained (Fig. 5e); a magnified FESEM image of these particles is shown in Fig. 5i. The micro-spheres in this sample were loose and oriented along the axial direction by rod-like particles 100-600 $\mathrm{nm}$ in size and spheroidal nanoparticles 50$100 \mathrm{~nm}$ in size. The formation of this novel micro/nanostructure relates to a non-classical crystallization mechanism called "oriented aggregation" which yields multiform crystal structures and morphologies. ${ }^{30}$

Analogical self-assembly structures were also reported in $\alpha$ $\mathrm{Fe}_{2} \mathrm{O}_{3}$ particles by Sugimoto et al. ${ }^{31}$ The rod-like particles in 

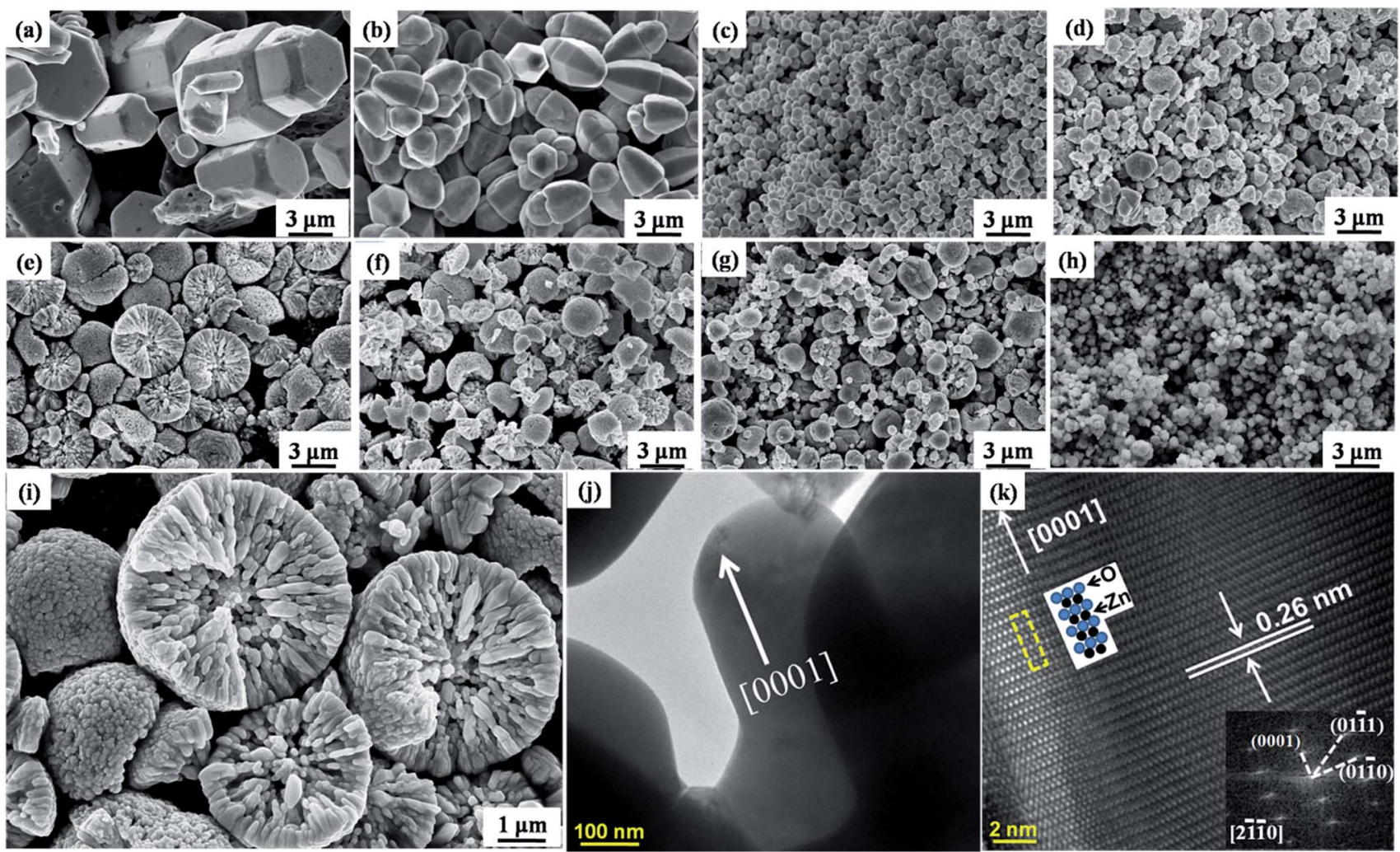

Fig. 5 FESEM micrographs of Al-free $\mathrm{ZnO}$ with TEA of $5 \mathrm{ml}$ (a), $10 \mathrm{ml}$ (b), $20 \mathrm{ml}$ (c), $25 \mathrm{ml}$ (d), $30 \mathrm{ml}$ (e and i) and $\mathrm{Zn} \mathrm{n}_{1-x} \mathrm{Al}_{x} \mathrm{O}$ using fixed TEA of $30 \mathrm{ml}$ at $x=0.01$ (f), $x=0.02(\mathrm{~g}), x=0.04(\mathrm{~h})$; TEM image (j); HRTEM image (k); inserted FFT diffractogram for the Al-free ZnO shown in (i).

micro/nano-structured ZnO powders (Fig. 5j) were around $c a$. $300 \mathrm{~nm}$ in diameter, which is consistent with the FESEM observation shown in Fig. 5i. The corresponding HRTEM analysis shown in Fig. 5k indicated that lattice spacing of $0.26 \mathrm{~nm}$ for the rod-like ZnO corresponded to the (0002) planes of the wurtzitestructured $\mathrm{ZnO}^{28}$ The fast Fourier transform (FFT) of the diffractogram (bottom, right corner) showed a single crystal-type [21̄10] zone-axis pattern. These results indicate that the growth direction of our highly crystalline $\mathrm{ZnO}$ rod-like particles was consistent with the crystallographic [0001], similar to the anisotropic growth of $\mathrm{ZnO}$ during $\mathrm{HS}^{32}$

Interestingly, the formation of this novel micro/nanostructure was hindered by adding $\mathrm{Al}$ as evidenced by the $x$ evolved morphology (Fig. 5f-h) when the TEA content was fixed to $30 \mathrm{ml}$. After doping Al to $x=0.01$, the loose micro/nanostructured powders shown in Fig. $5 \mathrm{f}$ decreased to $c a .3 \mu \mathrm{m}$ in micro-sphere diameter, to $c a .300 \mathrm{~nm}$ in rod-like particle size, and to $c a .40 \mathrm{~nm}$ in spheroidal particle size. This may have been because $\mathrm{Al}^{3+}$ acted as a competitive ligand of $\mathrm{Zn}^{2+}$ united with $\mathrm{OH}^{-}$to form $\mathrm{Al}(\mathrm{OH})_{3}$ prior $\left[\mathrm{Zn}(\mathrm{OH})_{4}\right]^{2-}$, which decreased the amount of $\left[\mathrm{Zn}(\mathrm{OH})_{4}\right]^{2-}$ dominating the anisotropic growth during the HS process. ${ }^{16,17}$ As $x$ increased to 0.02 and 0.04 (Fig. $5 \mathrm{~g}$ and $\mathrm{h}$ ), the sizes in particles and micro-spheres of micro/nanostructured powders further decreased. These results indicate that doping $\mathrm{Al}$ in the micro/nano-structured $\mathrm{ZnO}$ powders refined both the particles ${ }^{11}$ and the micro-spheres.
Phase and orientation of micro/nano-structured ZnO-based bulks

The micro/nano-structure of precursor powders shown in Fig. 6ad (enlarged in Fig. 6e-h) was clearly well-frozen in their corresponding bulks by applying SPS. The sizes of both micro-spheres and matrix grains also decreased as $x$ increased corresponding to the behavior of the precursor powders. Compared with the $x=$ 0 and $x=0.01$ bulks shown in Fig. $6 \mathrm{a}, \mathrm{b}$, e and $\mathrm{f}$, many white nanoprecipitates about $c a .10 \mathrm{~nm}$ in size were observed in the micro/nano-structured $x=0.02$ bulk in Fig. $6 \mathrm{c}$ and g, i.e., 10 to 20 times smaller than that of nano-structured bulks synthesized by Jood et al. ${ }^{11}$ and Nam et al. ${ }^{12}$ The white nanoprecipitates increased in quantity and average size to $c a .30 \mathrm{~nm}$ as $x$ increased to 0.04 in Fig. 6d and h. The EDX line analyses and atomic number contrast images (Fig. 6i) indicated that the new nanoprecipitates in Fig. $6 \mathrm{~g}$ and $\mathrm{h}$ were rich in $\mathrm{Al}$ and $\mathrm{O}$, corroborating the formation of $\mathrm{ZnAl}_{2} \mathrm{O}_{4}$. In addition to the different morphologies, the relative density of the micro/nano-structured bulks were $84 \%, 82 \%, 80 \%, 76 \%$, and $72 \%$ as $x=0,0.01,0.02,0.03$, and 0.04 , respectively, corresponding to increased quantities of pores. Overall, the refined matrix grains, $\mathrm{ZnAl}_{2} \mathrm{O}_{4}$ nanoprecipitates, and pores dominated the strong phonon scattering and contributes to the lower $k$ shown in Fig. 1c.

The phase structures of micro/nano-structured powders and bulks were compared as shown in Fig. 7a and b. The corresponding diffraction are well matched to those of wurtzite- 

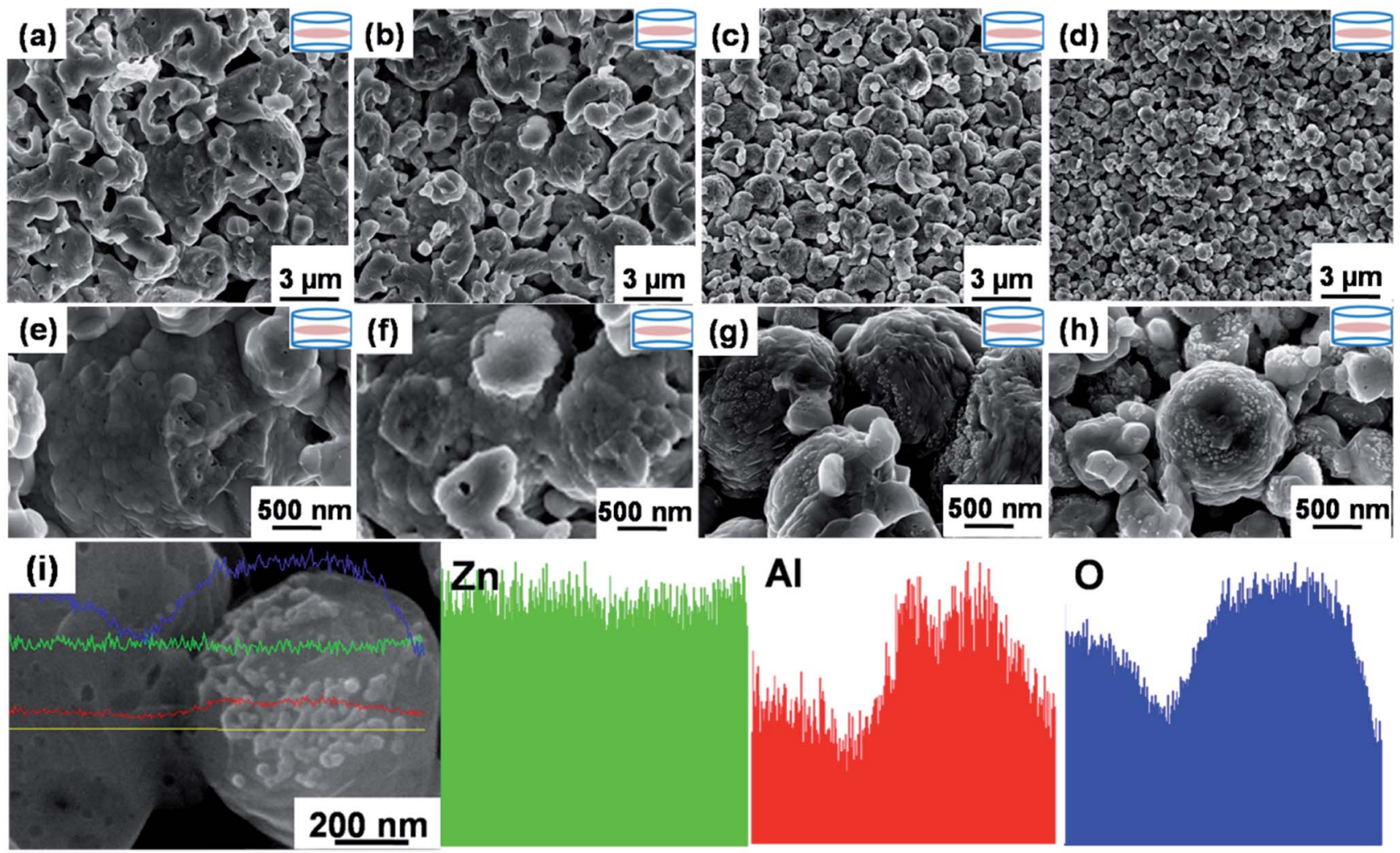

Fig. 6 FESEM micrographs of micro/nano-structured $\mathrm{Zn}_{1-x} \mathrm{Al}_{x} \mathrm{O}$ bulks with $x=0$ ( $\mathrm{a}$ and e), 0.01 (b and f), 0.02 (c and g) and 0.04 (d and h); representative EDX line scans of $\mathrm{O}, \mathrm{Zn}$, and $\mathrm{Al} \mathrm{K \alpha _{1 }} \mathrm{X}$-ray peaks obtained across $\mathrm{ZnAl}_{2} \mathrm{O}_{4}$ nanoprecipitates along yellow line in the $\mathrm{FESEM}$ micrograph (i) magnified from image (h)

structured $\mathrm{ZnO}$ (PDF\#36-1451). The secondary $\mathrm{ZnAl}_{2} \mathrm{O}_{4}$ impurity observed by FESEM micrographs in Fig. $6 \mathrm{~g}$ and $\mathrm{h}$ was undetected by XRD even as $x=0.04$, as their content was below the detection limit of the XRD analysis method. $\mathrm{ZnAl}_{2} \mathrm{O}_{4}$ impurity is typically detected by XRD in bulks sintered by solid state reaction (SSR), for example, in air at $1673 \mathrm{~K}$ for $10 \mathrm{~h}$ with $2 \%$ mol Al doping; ${ }^{10}$ but may be undetectable in the counterpart sintered by SPS below $1223 \mathrm{~K}$ for less than $10 \mathrm{~min}$ in a vacuum. ${ }^{12,33}$ We therefore speculated that the short sintering time (only $5 \mathrm{~min}$ ) and low sintering temperature (below $1223 \mathrm{~K}$ ) of SPS did not effectively facilitate the formation of $\mathrm{ZnAl}_{2} \mathrm{O}_{4}$.

The high solid solubility of Al contributing to the high $n$ (Fig. 2b) also contributed to the decreased formation $\mathrm{ZnAl}_{2} \mathrm{O}_{4}$. We did not observe any obvious trend in the main diffraction peaks in our bulks as compared to that of the powders shown in Fig. 7c, indicating delayed grain growth during the SPS process. This is also evidence that the micro/nano-structure of precursor powders was frozen perfectly in their corresponding bulks. Apart from the identical phase structure between powders and bulks, it is noted in Fig. 7a and b that the intensity of the (0002) diffraction peak differed as $x$ differed; this indicated a varied orientation caused by $x$ and the SPS process.

Fig. $7 \mathrm{~d}$ shows the orientation degree $F_{(000 t)}$ calculated by the Lotgering method ${ }^{34}$ for both powders and bulks as a function of $x$. The $F_{(000 l)}$ value is $10.8 \%$ for the micro/nano structured $x=$ 0 powder, which can be ascribed to the formation and growth of rod-like powders along the [0001] direction and the axially restriction by micro-spheres (Fig. 5i). This micro/nanostructural characteristic weakened as $x$ increased (Fig. 5e-h), which reflects the decreased $F_{(000 t)}$ value to $3.5 \%, 2.1 \%, 1.7 \%$, and $1.7 \%$ at $x=0.01, x=0.02,0.03$, and 0.04 . Compared to their corresponding powders, all bulks showed increased $F_{(000 t)}$ values to $21.8 \%, 6.4 \%, 9.7 \%, 2.7 \%$, and $4.2 \%$ as $x=0,0.01,0.02$, 0.03 , and 0.04 , respectively, indicating that the $F_{(000 l)}$ orientation in the powder was further enhanced in the corresponding bulk under applied pressure.

$\mathrm{ZnO}^{15,16,35}$ and $\mathrm{Bi}_{2} \mathrm{~S}_{3}{ }^{36}$ bulks have been reported to be preferentially oriented in the $(h k i 0)$ plane perpendicular $(\perp)$ to the pressure direction when rod-like powders grown preferentially at the same [0001] direction are consolidated, as rods readily collapse under applied pressure. Our bulks, which were preferentially oriented in $(000 l)$ perpendicular $(\perp)$ to the pressure direction, did not behave this way - we attribute this discrepancy to the micro/nano-structure having slowed the collapse rod-like particles due to spherical restrictions. The decreased $F_{(000 t)}$ with $x$ (Fig. 7d) in powders and bulks corresponds to decrease in both the diameter of micro-spheres as well as rod-like and spheroidal particles in the powders (Fig. 5e-h) or bulks (Fig. 6a-h) leading to fewer grain boundaries and less electron scattering. Because higher orientation degree relates to higher $\mu$, we assumed that the increased $\mu$ from $x=0$ to $x=0.01$ bulks (Fig. 2a) contributed predominantly to the reduced decree of misorientation among coherent grain boundaries rather than the $F_{(000)}$. Ultimately, we concluded that the $\mu$ values we obtained in the micro/nano- 

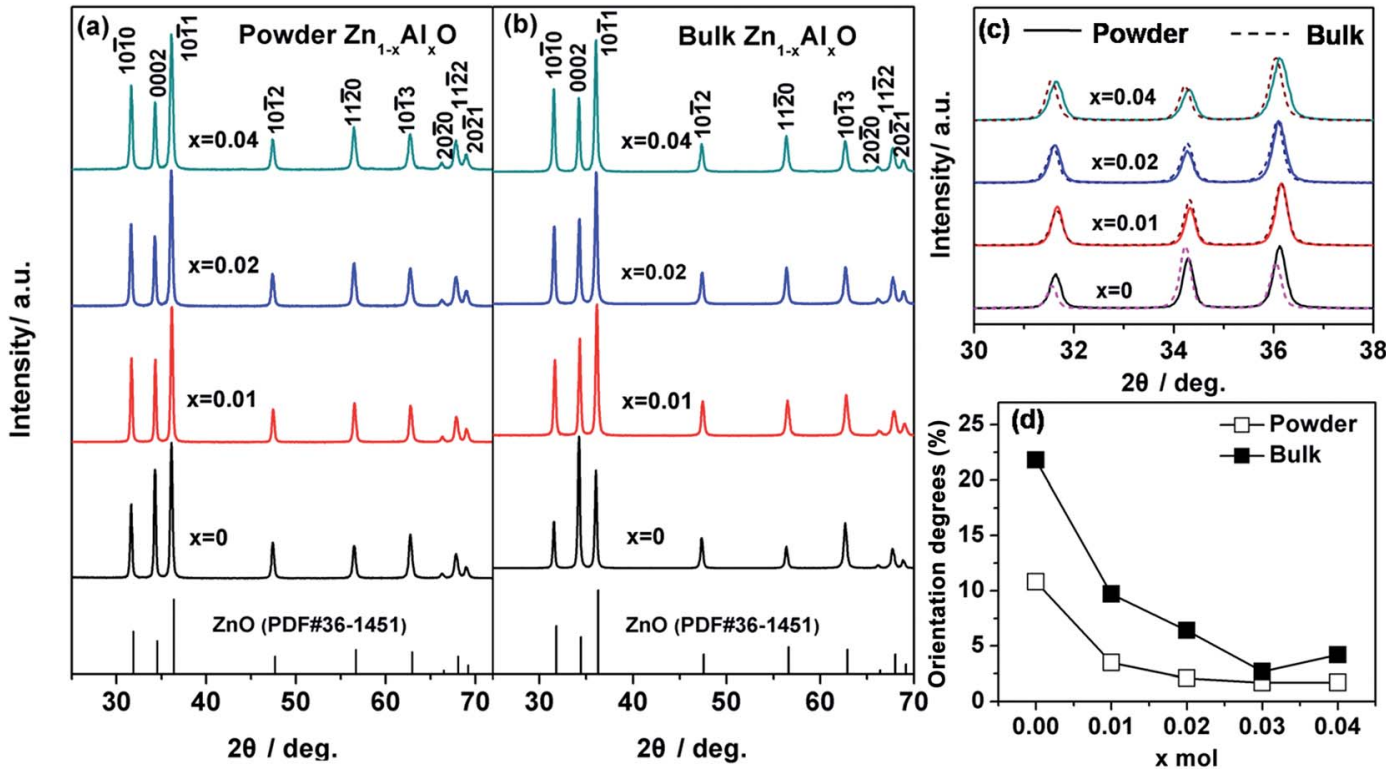

Fig. 7 XRD patterns in $25-70^{\circ}$ of $\mathrm{Zn}_{1-x} \mathrm{Al} \mathrm{l}_{x} \mathrm{O}(x=0,0.01,0.02,0.04)$ powders (a) and bulks (b) with respective XRD patterns in $30-38^{\circ}(\mathrm{c})$ and their corresponding texturing degrees $(\mathrm{d})$.

structured bulks were mainly related to the novel micro/nanostructure itself being conducive to the formation of a nearly coherent crystalline framework for rapid electron transfer.

\section{Conclusions}

A unique micro/nano structure was successfully obtained in $\mathrm{Zn}_{1-x} \mathrm{Al}_{x} \mathrm{O}(x=0,0.02,0.03,0.04 \mathrm{~mol})$ bulks prepared via $\mathrm{HS}$ and SPS. The nanograins were oriented along the axial direction of micro-spheres with a low misorientation degree between the neighboring grains. Due to the near-coherence of grain boundaries, the micro/nano structured $x=0.02$ bulk achieved a remarkably high $\mu$ value of $50.7 \mathrm{~cm}^{2} \mathrm{~V}^{-1} \mathrm{~s}^{-1}$ - close to that of single crystals - as well as a low $\kappa$ value $\left(2.1 \mathrm{~W} \mathrm{~m}^{-1} \mathrm{~K}^{-1}\right)$ likely arising in the nano-structured bulks due to effective scattering on the boundaries and interfaces. The synergistic optimization of electrical and thermal transport properties led to a high $Z T$ value, 0.36 , at $1073 \mathrm{~K}$.

The novel micro/nano-structure we established is likely applicable to other TE materials, as well. We also believe that this strategy can work in tandem with other successful TEenhancement approaches such as modulation doping and band structure engineering.

\section{Acknowledgements}

This work was supported by the National Natural Science Foundation of China (grant no. 51272023) and National Basic Research Program of China (grant no. 2013CB632503).

\section{References}

1 D. Kraemer, B. Poudel, H. P. Feng, J. C. Caylor, B. Yu, X. Yan, Y. Ma, X. W. Wang, D. Z. Wang, A. Muto, K. McEnaney,
M. Chiesa, Z. F. Ren and G. Chen, Nat. Mater., 2011, 10, 532538.

2 J. F. Li, W. S. Liu, L. D. Zhao and M. Zhou, NPG Asia Mater., 2010, 2, 152-158.

3 M. Zhou, J. F. Li and T. Kita, J. Am. Chem. Soc., 2008, 130, 4527-4532.

4 L. D. Zhao, B. P. Zhang, J. F. Li, M. Zhou, W. S. Liu and J. Liu, J. Alloys Compd., 2008, 455, 259-264.

5 J. Elias, M. Bechelany, I. Utke, R. Erni, D. Hosseini, J. Michler and L. Philippe, Nano Energy, 2012, 1, 696-705.

6 L. Li, T. Y. Zhai, Y. Bando and D. Golberg, Nano Energy, 2012, 1, 91-106.

7 L. Lin, Y. F. Hu, C. Xu, Y. Zhang, R. Zhang, X. N. Wen and Z. L. Wang, Nano Energy, 2013, 2, 75-81.

8 G. C. Yoon, K. S. Shin, M. K. Gupta, K. Y. Lee, J. H. Lee, Z. L. Wang and S. W. Kim, Nano Energy, 2015, 12, 547555.

9 M. Ohtaki, T. Tsubota, K. Eguchi and H. Arai, J. Appl. Phys., 1996, 79, 1816.

10 T. Tsubota, M. Ohtaki, K. Eguchi and H. Arai, J. Mater. Chem., 1997, 7, 85-90.

11 P. Jood, R. J. Mehta, Y. L. Zhang, G. Peleckis, X. L. Wang, R. W. Siegel, T. Borca-Tasciuc, S. X. Dou and G. Ramanath, Nano Lett., 2011, 11, 4337-4342.

12 W. H. Nam, Y. S. Lim, S. M. Choi, W. S. Seo and J. Y. Lee, J. Mater. Chem., 2012, 22, 14633-14638.

13 L. Han, N. Van Nong, W. Zhang, L. T. Hung, T. Holgate, K. Tashiro, M. Ohtaki, N. Pryds and S. Linderoth, RSC Adv., 2014, 4, 12353-12361.

14 D. S. Chen, Y. Zhao, Y. Chen, B. Wang, H. Y. Chen, J. Zhou and Z. Liang, ACS Appl. Mater. Interfaces, 2015, 7, 3224-3230.

15 T. Sahoo, M. Kim, J. H. Baek, S. R. Jeon, J. S. Kim, Y. T. Yu, C. R. Lee and I. H. Lee, Mater. Res. Bull., 2011, 46, 525-530. 
16 D. B. Zhang, B. P. Zhang, D. S. Ye and Y. B. Zhu, Ceram. Int., 2015, 41, 14627-14634.

17 D. B. Zhang, B. P. Zhang, D. S. Ye, Y. C. Liu and S. Li, J. Alloys Compd., 2016, 656, 784-792.

18 H. Zhang, D. Yang, Y. Ji, X. Y. Ma, J. Xu and D. L. Que, J. Phys. Chem. B, 2004, 108, 3955-3958.

19 H. Zhang, D. Yang, X. Y. Ma, Y. Ji, J. Xu and D. L. Que, Nanotechnology, 2004, 15, 622-626.

20 L. D. Zhao, X. Zhang, H. J. Wu, G. J. Tan, Y. L. Pei, Y. Xiao, C. Chang, D. Wu, H. Chi, L. Zheng, S. K. Gong, C. Uher, J. Q. He and M. G. Kanatzidis, J. Am. Chem. Soc., 2016, 138, 2366-2373.

21 J. G. Lu, Z. Z. Ye, Y. J. Zeng, L. P. Zhu, L. Wang, J. Yuan, B. H. Zhao and Q. L. Liang, J. Appl. Phys., 2006, 100, 73714.

22 D. C. Look, D. C. Reynolds, J. R. Sizelove, R. L. Jones, C. W. Litton, G. Cantwell and W. C. Harsch, Solid State Commun., 1998, 105, 399-401.

23 K. Ellmer, J. Phys. D: Appl. Phys., 2001, 34, 3097-3108.

24 W. H. Nam, B. B. Kim, S. G. Seo, Y. S. Lim, J. Y. Kim, W. S. Seo, W. K. Choi, H. H. Park and J. Y. Lee, Nano Lett., 2014, 14, 5104-5109.

25 F. May and G. J. Snyder, Introduction to Modeling Thermoelectric Transport at High Temperatures, in
Thermoelectrics and its Energy Harvesting, ed. D. M. Rowe, CRC Press, Boca Raton, 2012.

26 Y. He, P. Lu, X. Shi, F. F. Xu, T. S. Zhang, G. J. Snyder, C. Uher and L. D. Chen, Adv. Mater., 2015, 27, 3639-3644.

27 C. Xiao, J. Xu, B. X. Cao, K. Li, M. G. Kong and Y. Xie, J. Am. Chem. Soc., 2012, 134, 7971-7977.

28 S. Xu, Z. H. Li, Q. Wang, L. J. Cao, T. M. He and G. T. Zou, J. Alloys Compd., 2008, 465, 56-60.

29 R. G. Xie, D. S. Li, H. Zhang, D. R. Yang, M. H. Jiang, T. Sekiguchi, B. D. Liu and Y. S. Bando, J. Phys. Chem. B, 2006, 110, 19147-19153.

30 T. He, D. R. Chen, X. L. Jiao, Y. Y. Xu and Y. X. Gu, Langmuir, 2004, 20, 8404-8408.

31 T. Sugimoto, Y. S. Wang, H. Itoh and A. Muramatsu, Colloids Surf., A, 1998, 134, 265-279.

32 B. Cheng and E. T. Samulski, Chem. Commun., 2004, 8, 986.

33 K. H. Kim, S. H. Shim, K. B. Shim, K. Niihara and J. Hojo, J. Am. Ceram. Soc., 2005, 88, 628-632.

34 T. Tani, S. Isobe, W. S. Seo and K. Koumoto, J. Mater. Chem., 2001, 11, 2324-2328.

35 J. L. Ning, D. M. Jiang, K. H. Kim and K. B. Shim, Ceram. Int., 2007, 33, 107-114.

36 Z. H. Ge, B. P. Zhang, P. P. Shang and J. F. Li, J. Mater. Chem., 2011, 21, 9194-9200. 